\title{
TURBULENT, EXTREME MULTI-ZONE MODEL FOR SIMULATING FLUX AND POLARIZATION VARIABILITY IN BLAZARS
}

\author{
Alan P. Marscher \\ Institute for Astrophysical Research, Boston University, 725 Commonwealth Avenue, Boston, MA 02215, USA; marscher@ bu.edu \\ Received 2013 July 31; accepted 2013 November 19; published 2013 December 13
}

\begin{abstract}
The author presents a model for variability of the flux and polarization of blazars in which turbulent plasma flowing at a relativistic speed down a jet crosses a standing conical shock. The shock compresses the plasma and accelerates electrons to energies up to $\gamma_{\max } \gtrsim 10^{4}$ times their rest-mass energy, with the value of $\gamma_{\max }$ determined by the direction of the magnetic field relative to the shock front. The turbulence is approximated in a computer code as many cells, each with a uniform magnetic field whose direction is selected randomly. The density of high-energy electrons in the plasma changes randomly with time in a manner consistent with the power spectral density of flux variations derived from observations of blazars. The variations in flux and polarization are therefore caused by continuous noise processes rather than by singular events such as explosive injection of energy at the base of the jet. Sample simulations illustrate the behavior of flux and linear polarization versus time that such a model produces. The variations in $\gamma$-ray flux generated by the code are often, but not always, correlated with those at lower frequencies, and many of the flares are sharply peaked. The mean degree of polarization of synchrotron radiation is higher and its timescale of variability shorter toward higher frequencies, while the polarization electric vector sometimes randomly executes apparent rotations. The slope of the spectral energy distribution exhibits sharper breaks than can arise solely from energy losses. All of these results correspond to properties observed in blazars.
\end{abstract}

Key words: BL Lacertae objects: general - galaxies: active - galaxies: jets - polarization - quasars: general

Online-only material: color figure

\section{INTRODUCTION}

The blazar class of active galactic nuclei is characterized by extreme variability of flux and polarization of nonthermal radiation across the electromagnetic spectrum. Statistical analyses of the flux variations-in particular, the power-law power spectra (Chatterjee et al. 2008, 2009, 2012; Abdo et al. 2011)—imply that the variations are governed by noise processes with higher amplitudes on longer timescales. Furthermore, the linear polarization of blazars ranges from a few to tens of percent and tends to be highly variable in both degree and position angle (e.g., D'Arcangelo et al. 2007; Marscher et al. 2010; Itoh et al. 2013). Although the range of degree of polarization and the tendency for the position angle to be similar to or nearly transverse to the jet axis (e.g., Jorstad et al. 2007) can be explained with a helical magnetic field (Lyutikov et al. 2005; Pushkarev et al. 2005), the rapid variations in polarization are not naturally reproduced in a model in which the field is either $100 \%$ globally ordered or completely chaotic on small scales. In the former case, the ordered field provides stability to the polarization, while in the latter case the polarization cancels in the presence of an essentially infinite number of random field directions.

A natural explanation for the noise-like fluctuations of flux and polarization in blazars is the presence of turbulence in the relativistic jets that emit the nonthermal radiation. If one approximates the pattern of the turbulence in terms of $N$ cells, each with a uniform magnetic field with random orientation, the degree of linear polarization has a mean value of $\langle\Pi\rangle \approx \Pi_{\max } N^{-1 / 2}$, where $\Pi_{\max }$, typically between 0.7 and 0.8 , corresponds to the uniform field case (Burn 1966). If the cells pass into and out of the emission region, the polarization will vary about the mean with a standard deviation $\sigma_{\Pi} \approx 0.5 \Pi_{\max } N^{-1 / 2}$ (Jones 1988). The electric-vector position angle (EVPA) $\chi$ of the polarization will also change with time in a random manner that can often appear as a rotation over hundreds of degrees (Jones 1988; Marscher et al. 2008; D’Arcangelo 2009).

The superposition of ordered and turbulent magnetic field components can provide the combination of a preferred orientation of the polarization vector plus fluctuations in $\Pi$ and $\chi$. Here the author introduces a numerical model that creates this combination by passing turbulent cells through a standing shock wave. Daly \& Marscher (1987), Cawthorne \& Cobb (1990), Gómez et al. (1995), and D'Arcangelo et al. (2007) have proposed that a stationary conical shock caused by a pressure mismatch with the external medium corresponds to the bright compact structure (the "core") observed at the upstream end of blazar jets imaged with very long baseline interferometry (VLBI) at millimeter wavelengths. In this spectral range, relatively low optical depths allow jets of many blazars to be viewed on sub-parsec scales with minimal obscuration by synchrotron self-absorption.

The following two sections describe the physical model and a numerical code that implements the model. This "turbulent extreme multi-zone" (TEMZ) code calculates, as a function of time, the spectral energy distribution (SED) from synchrotron radiation and inverse Compton (IC) scattering, as well as the linear polarization of the synchrotron emission at various frequencies. Seed photons for the scattering include infrared (IR) emission by hot dust in a parsec-scale molecular torus, as well as synchrotron and IC photons from a Mach disk (MD) on the jet axis. The MD, often called a "working surface" when it occurs near the end of a jet, is a strong shock oriented transverse to the jet axis that forms near the apex of a conical shock when the jet maintains azimuthal symmetry (Courant \& Friedrich 1976).

After the description of the model in Section 2, Section 3 briefly reviews the tests carried out to check the accuracy of the numerical code. Section 4 presents the results of a small number of numerical simulations that serve as illustrations of 


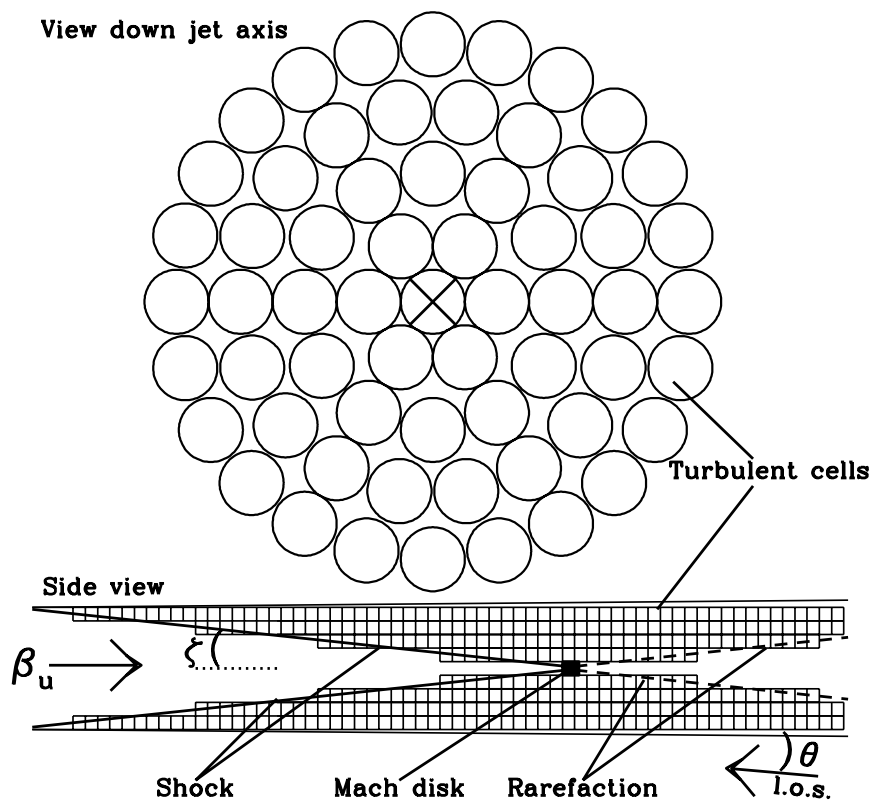

Figure 1. Sketch of the geometry employed to carry out calculations within the TEMZ code. The number of fixed computational cells across the jet crosssection (view down axis, in which $\times$ marks the Mach disk) can be as high as 1140 , not including the Mach disk. A turbulent cell of plasma, moving at laminar velocity $\beta_{u}$ upstream of the shock and at laminar velocity $\beta_{d}$ after it passes the shock, crosses one computational cell during each time step. The emission occurs between the conical standing shock and the rarefaction. The entire region sketched lies $\gtrsim 1 \mathrm{pc}$ from the central engine.

the variability patterns that the model generates, while Section 5 presents the conclusions of this work. A future study will include a more complete exploration of parameter space.

\section{DESCRIPTION OF THE TEMZ MODEL AND NUMERICAL CODE}

The author has developed a numerical code designed to simulate the time-dependent SED from a relativistic jet in which turbulent plasma crosses a standing conical shock plus MD. (The code, originally written in the Fortran-77 computer language, has also been translated to the $\mathrm{C}++$ language by $\mathrm{M}$. Valdez.) The run time on a common desktop or laptop computer with a Linux operating system is hours or days, depending on the number of emission zones ("cells"), values selected for the physical parameters, and the number of simulated time steps involved (hundreds to thousands). The program calculates the SED from $1 \times 10^{10}$ to $5.6 \times 10^{26} \mathrm{~Hz}$, with four frequencies per decade, as well as the degree and position angle of linear polarization (for synchrotron radiation only) at each time step.

As depicted in Figure 1, the TEMZ geometry consists of many cylindrical computational cells, fixed in space, through each of which a single turbulent cell of plasma passes during one time step. The plasma contains magnetic field and relativistic electrons (and positrons, if any), as well as protons that are presumed not to contribute a significant amount of radiation. The field is uniform within any given turbulent cell. The plasma flows downstream, i.e., radially away from the central engine, through the computational cells at a laminar relativistic velocity $\beta_{\mathbf{d}} c$, where $c$ is the speed of light and the subscript "d" refers to the region downstream of the shock. The corresponding bulk Lorentz factor is defined as $\Gamma_{d} \equiv\left(1-\beta_{d}^{2}\right)^{-1 / 2}$. The plasma within each turbulent cell has a turbulent component $\beta_{\mathbf{t}} c$ of the bulk velocity, with a randomly selected direction relative to the systematic flow. For simplicity, and because of the high Lorentz factor at which the fluid is advected downstream, the turbulent component of the bulk velocity is subsequently held constant. Also for simplicity, the model approximates that there is no physical interaction between adjacent cells.

The radiating electrons (as well as any positrons) are accelerated to relativistic energies as the plasma crosses a conical "recollimation" shock, oriented such that it narrows with distance from the central engine. An MD can be present at the apex of the conical shock. Beyond the apex, the flow crosses a conical rarefaction that causes the flow to expand and accelerate. The current version of the code assumes that the emission turns off after the plasma crosses the rarefaction. While this may not be the case at lower frequencies given the long timescales for energy losses by the radiating electrons, the assumption lowers the computing time considerably. Hence, the accuracy of the results presented here may decrease toward centimeter wavelengths, where the flux density is generally higher than that calculated in the TEMZ code. In many blazars, the flux at such wavelengths is dominated by features well downstream of the "core" structure treated by the TEMZ model.

Figure 1 sketches the geometry, as viewed both down the axis and from the side, of the section of the jet over which the calculations are performed. (Note that the geometry of the computational cell structure is approximated to be cylindrical. This ignores the slight spreading of the jet expected from the small transverse components of both the laminar and turbulent velocities. These components are included in the calculation of Doppler factors described below, however.) The time step, in the observer's frame, is selected as the time required for a turbulent cell of plasma to move downstream by one computational cell length $\ell=0.2 R_{\text {cell }} / \tan \zeta$, where the cell cross-sectional radius $R_{\text {cell }}$ is selected by the user and $\zeta$, also a free parameter (although subject to constraints; see below), is the angle between the conical shock front and the jet axis. The factor of 0.2 corresponds to 2 divided by the number of cells (10) in an outer column (where the "columns" are parallel to the jet axis) between the start of that column and the start of the neighboring column (see Figure 1).

The energy density of relativistic electrons $u_{e}$ in the upstream, unshocked plasma fluctuates about a nominal value $u_{e}^{*}$ set by the user, modulated randomly with time within a distribution that reproduces a power spectral density (PSD) of flux variations with a power-law shape. A subroutine supplied by R. Chatterjee (see Chatterjee et al. 2008), based on the algorithm of Timmer $\&$ König (1995), produces $2^{n}$ random values between -1 and 1 that correspond to a power-law PSD with a slope provided by the user, where $n$ is an integer. Following a suggestion by Uttley et al. (2005), the TEMZ code exponentiates each number produced by the subroutine in order to derive the multiplicative factor that is applied to the input value of the energy density. (A new, improved prescription by Emmanoulopoulos et al. 2013, for producing the fluctuations will be incorporated in the future.) This is done for $2^{17}=131,072$ times, which is sufficient to allow calculations over the desired number of time steps included in the light curves while taking into account lighttravel delays across the grid of cells. The code averages the fluctuations that are thereby produced over 10 time steps, an action that is necessary because of the discreteness of the cell arrangement (see the previous paragraph). (The current version of the code includes gradients in unshocked energy density only in the longitudinal direction (through the time dependence), not in the transverse direction.) For the same reason, the orientation 
of the unshocked magnetic field vector is selected at random every 10 time steps, i.e., for every tenth turbulent cell along a given column. Within the intermediate cells, the unit vector defining the direction of the field is rotated smoothly between the previous and next randomly selected directions, along either the longest or shortest path of rotation (selected randomly with $50 \%$ probability for each). This procedure smooths the discrete changes in field direction and prevents unphysical discontinuities in the magnetic field vector along any given column. (There is, however, no relation between the directions of the unshocked magnetic field lines in adjacent cells of different columns in the current version of the code.) The magnetic field strength $B$ is determined via an assumption that the upstream magnetic energy density is a constant fraction $f_{\mathrm{B}}$ of the nominal electron energy density $u_{e}^{*}$,

$$
u_{B}=B^{2} /(8 \pi)=f_{B} u_{e}^{*} .
$$

(The code contains an option, not implemented in the calculations presented here, that allows the magnetic energy density to fluctuate with time such that it is always proportional to $u_{e}$.) All energy densities, magnetic fields, electron energies, and electron energy distributions are evaluated in the plasma rest frame unless otherwise indicated.

At every time step, electrons are injected into the cells immediately beyond the shock. The injection is in the form of a power-law energy distribution with slope $-p$ and low-energy cutoff $\gamma_{0 \text {,min }}$, both chosen by the user. The high-energy cutoff $\gamma_{0, \max }$ falls within a range from $\gamma_{\text {max,low }}^{*}$ to $\gamma_{\text {max,high }}^{*}$ also set by the user, but with a value within this range that is either: (1) equal to $\gamma_{\text {max,high }}^{*}\left(B_{\|} / B\right)^{2}$ (but not less than $\gamma_{\text {max,low }}^{*}$ ), where the subscript $\|$ indicates the component of the magnetic field that is parallel to the shock normal; or (2) selected at random within a power-law distribution (with slope as an input parameter). The first option, selected for the calculations presented below, is meant to represent in a crude manner the higher efficiency of particle acceleration by shocks when $\mathbf{B}$ is nearly parallel to the shock normal and when plasma turbulence is mild. The latter means that the scattering length of electron motions is too long for particles to cross the shock front many times when the field is more inclined to the shock normal (see, e.g., Summerlin \& Baring 2012, for simulations and a discussion related to this).

The compression ratio $\eta$ of the conical standing shock follows the expression given by Cawthorne \& Cobb (1990), which is derived from Lind \& Blandford (1985):

$$
\eta=\Gamma_{u} \beta_{u} \sin \zeta\left(8 \beta_{u}^{2} \sin ^{2} \zeta-\Gamma_{u}^{-2}\right)^{1 / 2}\left(1-\beta_{u}^{2} \cos ^{2} \zeta\right)^{-1 / 2} .
$$

Here, $\beta_{u}$ and $\Gamma_{u}$ are the speed in light units and Lorentz factor, respectively, of the bulk flow upstream of the shock, and $\eta$ is defined as the ratio of the density of the shocked to unshocked plasma. (Note that the latter is the inverse of the definition used by Cawthorne \& Cobb 1990.) In order to satisfy the criterion for a shock, the angle $\zeta$ that the shock front subtends to the jet axis must satisfy the criterion $\sin \zeta>\left(\sqrt{2} \beta_{u} \Gamma_{u}\right)^{-1}$ for an ultra-relativistic equation of state. On the other hand, if the value of $\zeta$ is too large, the shock decelerates the flow so much (the downstream velocity component parallel to the shock normal drops to $\beta_{d, \|}=1 / 3$ ) that the effects of relativistic beaming become weak, contrary to the inference that beaming of the emission is particularly strong in blazars.

The shock compresses the component of the magnetic field that is perpendicular to the shock normal by a factor of $\eta$. The magnetic field $\mathbf{B}$ is calculated in the plasma rest frame, which changes after the plasma crosses the shock. In order to calculate the downstream value of $\mathbf{B}$, the code transforms the upstream field to the standing shock frame (which is the same as the rest frame of the host galaxy), applies the compression factor to the component perpendicular to the shock normal, and then transforms the field to the rest frame of the downstream plasma (see Lyutikov et al. 2003).

\subsection{Evolution of the Electron Energy Distribution Downstream of the Shock}

After the power-law injection of relativistic electrons as plasma crosses the shock front, the code follows the evolution of the energy distribution as the electrons lose energy via radiative losses. The jet opening angle $\phi$ is assumed to be so small (see Jorstad et al. 2007; Pushkarev et al. 2012) that expansion cooling is not important within the section of the jet under consideration. The code employs the standard formula (e.g., Pacholczyk 1970) for the decrease of energy (in rest-mass units) $\gamma$ with time:

$$
\dot{\gamma}=-k_{r}\left(B^{2}+8 \pi u_{\mathrm{ph}}\right) \gamma^{2}
$$

where $k_{r}=1.3 \times 10^{-9} \mathrm{erg}^{-1} \mathrm{~s}^{-1} \mathrm{~cm}^{3}$. Here, $u_{\mathrm{ph}}$ is the energy density of seed photons that are subject to IC scattering off the electrons. The formula assumes that scattering of the electrons off magnetic irregularities randomizes the pitch angles many times over the energy-loss timescale, and that the field of seed photons is isotropic in the rest frame of the plasma. The code calculates $u_{\mathrm{ph}}$ for each cell at each time step, but only includes photon energies that fall below the Klein-Nishina limit, beyond which the scattering efficiency decreases such that the energy losses are much lower than given by the above equation. Both this approximation and the isotropy assumption lead to a modest sacrifice in accuracy while allowing an analytical calculation of the electron energy spectrum as a function of time, which greatly reduces the time required for computations. The seed photons arriving at a given cell comprise both the steady source of IR emission from a dust torus and time-variable emission from the MD. Calculation of the latter is carried out for an earlier time, when the photons arriving at the cell left the MD.

Since the plasma advects beyond the shock with a velocity $\beta_{d} \approx 1$, the distance $z_{\text {loss }}(\gamma)$ beyond the shock that contains electrons of energy $\gamma$ is inversely related to $\gamma$ :

$$
z_{\text {loss }}(\gamma)=\left(7.5 \mathrm{erg} \mathrm{cm}^{-3}\right)\left(B^{2}+8 \pi u_{\mathrm{ph}}\right)^{-1}\left[\gamma^{-1}-\gamma_{0, \max }^{-1}\right] \Gamma_{d} \mathrm{pc}
$$

where the last term accounts for length contraction in the plasma frame (Marscher \& Gear 1985). This equation can be inverted to find the maximum energy $\gamma_{\max }(z)$ retained by electrons after they cross a distance $\left(z-z_{s}\right)_{\mathrm{pc}}$ beyond the shock front, which is located at longitudinal position $z_{s}$ :

$$
\begin{aligned}
& \gamma_{\max }(z)= \\
& \frac{\gamma_{0, \max }}{1+\left(0.13 \mathrm{erg}^{-1} \mathrm{~cm}^{3}\right)\left(B^{2}+8 \pi u_{\mathrm{ph}}\right)\left(z-z_{s}\right)_{\mathrm{pc}} \Gamma_{d}^{-1} \gamma_{0, \max }} .
\end{aligned}
$$

A similar equation for the lowest electron energy replaces $\gamma_{\max }(z)$ by $\gamma_{\min }(z)$ and $\gamma_{0, \max }$ by $\gamma_{0, \min }$.

The electron energy distribution within a cell follows the formula for steady injection of electrons into a volume while 
radiative losses are operating (Kardashev 1962; Pacholczyk 1970):

$$
N(\gamma, z)=Q_{0} \gamma^{-p} \int_{0}^{t}\left[1-k_{r}\left(B^{2}+8 \pi u_{\mathrm{ph}}\right) t^{\prime} \gamma\right]^{p-2} d t^{\prime},
$$

where the injected electron energy distribution is a power-law of slope $-p$, and $Q_{0}$, which is related to $u_{e}$, defines the injection rate. Here, the upper limit $t$ is the lesser of $\left(z-z_{s}\right)\left(\Gamma_{d} \beta_{d} c\right)^{-1}$ and $\left[\left(\gamma_{0, \max } / \gamma\right)-1\right]\left[k_{r}\left(B^{2}+8 \pi u_{\mathrm{ph}}\right) \gamma_{0, \max }\right]^{-1}$. For a single cell with injection at one end, the integral can be evaluated as:

$$
\begin{aligned}
N(\gamma, z)= & Q_{0}\left[(p-1) k_{r}\left(B^{2}+8 \pi u_{\mathrm{ph}}\right)\right]^{-1} \gamma^{-(p+1)} \\
& \times\left\{1-\left[1-k_{r}\left(B^{2}+8 \pi u_{\mathrm{ph}}\right) t \gamma\right]^{p-1}\right\}
\end{aligned}
$$

This equation is valid for $\gamma_{0, \min } \leqslant \gamma \leqslant \gamma_{\max }(z)$ and only for plasma in computational cells that border the standing shock. For all cells farther downstream than this, the code takes into account the change in seed photon energy density with time in a given turbulent cell of plasma as it propagates through the computational cells downstream of the shock. That is, the value of $u_{\mathrm{ph}}$ used in Equation (7) is the average since the time that the plasma crossed the shock.

In the MD, where the magnetic field and density of electrons are much higher than in the plasma that crosses the conical shock, the electrons are in the "fast cooling regime," i.e., they radiate most of their energy before crossing one cell length. The ratio of the photon to magnetic energy density adopted to compute the energy losses of electrons in the MD plasma follows that calculated by Sari $\&$ Esen (2001), $u_{\mathrm{ph}}=\varepsilon B^{2} /(8 \pi)$, where $\varepsilon=0.5\left[\sqrt{1+\left(4 / f_{B}\right)}-1\right]$.

\subsection{Calculation of Synchrotron Emission and Absorption}

The code computes the synchrotron emission coefficient $j_{v}$ within each cell at every time step via the standard formula (e.g., Pacholczyk 1970)

$$
j_{v}^{S}\left(\nu^{\prime}\right)=\frac{\sqrt{3} e^{3}}{4 \pi m c^{2}} B \sin \psi \int_{\gamma_{\min }(z)}^{\gamma_{\max }(z)} N(\gamma, z) \mathcal{F}\left(\nu^{\prime} / \nu_{c}\right) d \gamma
$$

Here, $v^{\prime}$ is the frequency of the emission in the plasma rest frame, $e$ is the magnitude of the electron charge, $m$ is the electron rest mass, $\psi$ is the angle between the magnetic field and the (aberrated) line of sight in the plasma frame, and $v_{c}=2 e B \gamma^{2} /(4 \pi m c)$ is the synchrotron critical frequency, averaged over electron pitch angle. The function $\mathcal{F}\left(v^{\prime} / v_{c}\right)$ is the usual synchrotron kernel, given in Pacholczyk (1970), for example. The code uses the excellent approximation for $\mathcal{F}\left(v^{\prime} / v_{c}\right)$ found by Joshi \& Böttcher (2011).

The angle $\psi$ is computed by Lorentz transforming the line-ofsight unit vector $\hat{\mathbf{s}}$ to the plasma (primed) frame. The coordinates are selected such that $\hat{\mathbf{s}}$ lies in the $\hat{\mathbf{x}}-\hat{\mathbf{z}}$ plane. The value of $B \sin \psi$ is then calculated as $\left|\hat{\mathbf{s}}^{\prime} \times B\right|$.

The TEMZ code calculates the absorption coefficient in each cell in a similar manner, again using a standard expression (e.g., Pacholczyk 1970):

$$
\begin{aligned}
\kappa_{\nu}^{S}\left(v^{\prime}\right)= & \frac{(p+2) \sqrt{3} e^{3}}{4 \pi m c^{2}} B \sin \psi \\
& \times \int_{\gamma_{\min }(z)}^{\gamma_{\max }(z)} N(\gamma, z) \gamma^{-1} \mathcal{F}\left(v^{\prime} / \nu_{c}\right) d \gamma .
\end{aligned}
$$

The intensity of radiation leaving the cell follows the usual solution to radiation transfer in a uniform source: $I_{v}\left(v^{\prime}\right)=$ $\left[j_{v}\left(v^{\prime}\right) / \kappa_{v}\left(v^{\prime}\right)\right]\left\{1-\exp \left[-\tau_{v}\left(v^{\prime}\right)\right]\right\}$, where $\tau_{v}$ equals $\kappa_{v}$ times the path length through the cell, which is equal to the cell length to a very good approximation for small angles to the line of sight $\theta_{\mathrm{los}}$. If there are $n_{\mathrm{ext}}$ other cells along the line of sight between the cell of interest and the observer, the intensity is attenuated by a factor of $\exp \left[-n_{\operatorname{ext}} \tau_{v}\left(v^{\prime}\right)\right]$. This approximation is employed to conserve computation time. It is roughly valid since the electrons responsible for absorption have low energies, and thus are not strongly affected by radiative energy losses. (A more accurate calculation of absorption is under development.) The flux density from a cell equals the intensity of radiation received from the cell times the essentially circular area of its face. The total flux density $F_{v}$ from the source is a simple sum over that from all cells.

\subsection{Calculation of Inverse Compton Emission}

For a given spectral intensity $I_{v^{\prime}}$ of seed photons arriving at a cell, the emission coefficient of IC scattering requires a double integration over electron energy and seed photon frequency. While it also properly involves a double integral over photon angles, this requires excessive computing time when many emission zones are involved. The code therefore utilizes the Klein-Nishina cross-section derived by Dermer \& Menon (2009; Equation (6.31)) under the "head-on" approximation that the photon is scattered in the initial direction of the electron's motion:

$$
\sigma_{\mathrm{KN}}=\frac{3}{8} \sigma_{T}\left(y^{\prime}+y^{\prime-1}-2 q^{\prime}+q^{\prime 2}\right) /\left(\gamma \epsilon_{i}^{\prime}\right),
$$

where $y^{\prime} \equiv 1-\left(\epsilon^{\prime} / \gamma\right), q^{\prime} \equiv \epsilon^{\prime} /\left(\gamma y^{\prime} \epsilon_{i}^{\prime}\right)$, and $\epsilon^{\prime} \equiv h v^{\prime} /\left(m c^{2}\right)$, $v_{i}^{\prime}$ is the frequency of the seed photon, $v^{\prime}$ is the frequency of the scattered photon, $\sigma_{T}=6.653 \times 10^{-25} \mathrm{~cm}^{2}$ is the Thomson cross-section, and $h$ is Planck's constant. The cross-section is valid for $\left(1+4 \gamma \epsilon_{i}^{\prime}\right)^{-1}<y^{\prime}<1$.

The IC emission coefficient is then

$$
j_{v}^{\mathrm{IC}}\left(v^{\prime}\right)=\int_{\gamma_{1}}^{\gamma_{\max }} \int_{v_{i, 1}^{\prime}}^{\infty} I_{\text {seed }}^{\prime}\left(v_{i}^{\prime}\right) N(\gamma, z) \sigma_{\mathrm{KN}}\left(v^{\prime}, v_{i}^{\prime}, \gamma\right) d v_{i}^{\prime} d \gamma,
$$

where $\gamma_{1}$ is the greater of $\gamma_{\min }$ and $\epsilon^{\prime}\left[1+\sqrt{1+\left(\epsilon_{i}^{\prime} \epsilon^{\prime}\right)^{-1}}\right] / 2$, $v_{i, 1}^{\prime}=v^{\prime}\left[4 \gamma^{2}\left(1-\epsilon^{\prime} \gamma^{-1}\right)\right]^{-1}$, and $I_{\text {seed }}^{\prime}\left(\nu_{i}^{\prime}\right)$ is the spectral intensity of seed photon emission as measured in the plasma rest frame.

Equation (10) contains an implicit dependence on the angle between the bulk velocity vector of the plasma and the direction of the source of seed photons. This results from the Doppler formula for the transformation of frequency from the rest frame of the seed photon source (SP) to the plasma frame: $v_{i}^{\prime}=v_{i} \delta_{\mathrm{SP}}$, where

$$
\delta_{\mathrm{SP}}=\left[\Gamma_{\text {rel }}\left(1-\beta_{\text {rel }} \cos \vartheta_{\text {rel }}^{\prime}\right]^{-1} .\right.
$$

Here, $\beta_{\text {rel }}$ is the magnitude of the relative velocity in light units between the plasma and seed photon source, $\Gamma_{\text {rel }}$ is the corresponding Lorentz factor, and $\vartheta_{\text {rel }}^{\prime}$ is the aberrated angle between the velocity vector of the plasma and the aberrated direction of the seed photon source in the plasma frame.

\subsection{Sources of Seed Photons for Inverse Compton Scattering}

The current version of the TEMZ code includes two sources of seed photons available for IC scattering by electrons in the 
Table 1

Adjustable Parameters in the TEMZ Computer Code

\begin{tabular}{|c|c|c|c|}
\hline Symbol & Description & Range of Values & Notes \\
\hline$n_{\mathrm{rad}}$ & Number of cells between Mach disk and jet boundary & $4-19$ & Cross-section contains $3\left(n_{\mathrm{rad}}+1\right) n_{\mathrm{rad}}$ cells \\
\hline$Z$ & Redshift & $\ldots$ & From observations of blazar of interest \\
\hline$z_{\mathrm{MD}}$ & Distance of Mach disk/shock apex from central engine & $1-20 \mathrm{pc}$ & \\
\hline$p$ & -1 times slope of injected electron energy distribution & $1.5-3$ & Single power-law assumed in absence of radiative losses \\
\hline$-b$ & Power-law slope of power spectral density & $1.5-2$ & Used to determine modulation of energy density \\
\hline$B$ & Unshocked magnetic field & $0.01-0.5$ & Modulated as square-root of energy density \\
\hline$f_{B}$ & Ratio of magnetic to relativistic electron energy density & $0.01-1$ & Equipartition value is $\sim 1$ \\
\hline$R_{\text {cell }}$ & Radius of a cylindrical cell & $0.002-0.05 \mathrm{pc}$ & Depends on scale size and value of $n_{\text {rad }}$ \\
\hline$\gamma_{0, \min }$ & Lowest energy of electrons accelerated by shock & $10-2000$ & Low-energy cutoff of power-law injection \\
\hline$\gamma_{\max , \text { high }}^{*}$ & Highest possible value of $\gamma_{0, \max }$ produced by shock & $5000-10^{6}$ & Optimal acceleration of electrons by the shock \\
\hline$\gamma_{\max , \text { low }}^{*}$ & Lowest value of $\gamma_{0, \max }$ produced by shock & $1000-10^{4}$ & Lowest efficiency of acceleration \\
\hline$\beta_{\mathbf{u}}$ & Bulk laminar velocity of unshocked plasma in light units & $0.97-0.9998$ & \\
\hline$\beta_{\mathbf{t}}$ & Turbulent velocity of unshocked plasma in light units & $0-0.577$ & Range assumes subsonic turbulence \\
\hline$\zeta$ & Angle between conical shock and jet axis & $4^{\circ}-10^{\circ}$ & Must satisfy criterion $\sin \zeta>\left(\sqrt{2} \beta_{u} \gamma_{u}\right)^{-1}$ \\
\hline$\theta_{\mathrm{los}}$ & Angle between jet axis and line of sight & $0^{\circ}-20^{\circ}$ & \\
\hline$\phi$ & Opening semi-angle of jet & $0^{\circ}-3^{\circ}$ & \\
\hline$A_{\mathrm{MD}}$ & Ratio of cross-sectional areas of Mach disk and a cell & $0.01-10$ & \\
\hline$T_{\text {dust }}$ & Blackbody temperature of hot dust torus & $1000-1200 \mathrm{~K}$ & Observed value in two blazars \\
\hline$L_{\text {dust }}$ & Luminosity of thermal IR emission from hot dust & $1 \times 10^{44}-2 \times 10^{46} \mathrm{erg} \mathrm{s}^{-1}$ & \\
\hline$r_{\text {dust }}$ & Mean distance of dust torus from black hole & $1-5 \mathrm{pc}$ & \\
\hline$R_{\text {dust }}$ & Cross-sectional radius of dust torus & $0.2-0.5 r_{\text {dust }}$ & \\
\hline
\end{tabular}

cells: IR blackbody emission from hot dust contained in a patchy molecular torus (external Compton, hereafter "EC-Dust") and synchrotron plus first-order synchrotron self-Compton emission from a Mach disk ("SSC-MD") centered on the jet axis at the narrow end of the conical shock (see Figure 1). Because of the relativistic motion of the plasma in the cells, the radiation from both of these sources is blueshifted and beamed in the plasma rest frame. The code does not currently include the seed photons from synchrotron emission within the cell under consideration ("SSC") plus all of the other cells ("SSC-C"), owing to the excessive computational time required given the large number of cells. Future parallelization of the $\mathrm{C}++$ version of the code is planned in an effort to make such calculations possible.

The central circle of the dusty torus lies a distance $r_{t}$ from the center of the system, assumed to be a black hole $(\mathrm{BH})$, and the torus has a cross-sectional radius $R_{t}$. As viewed from a cell at a polar distance $z$ from the $\mathrm{BH}$, the dusty torus subtends a ring in the sky. In the rest frame of the system, the direction of the outer/inner boundary of the ring subtends an angle

$$
\xi_{\text {in }}^{\text {out }} \approx \tan ^{-1}\left(r_{t} / z\right) \pm \sin ^{-1}\left[R_{t}\left(z^{2}+r_{t}^{2}\right)^{-1 / 2}\right]
$$

to the direction toward the $\mathrm{BH}$. In the rest frame (primed) of the plasma in the cell, which moves at velocity $\beta_{\mathbf{d}}$ (Lorentz factor $\Gamma_{d}$ ) approximately in the $\hat{\mathbf{z}}$ direction (the slight deviation from this direction is unimportant for this calculation), each of these angles is transformed as

$$
\tan \left(\xi^{\prime} / 2\right)=\tan (\xi / 2)\left[\Gamma_{d}\left(1+\beta_{d}\right)\right]^{-1} .
$$

The blackbody emission from the torus in the plasma frame corresponds to a temperature

$$
T^{\prime}=\delta_{\mathrm{SP}} T=\left[\Gamma_{d}\left(1-\beta_{d} \cos \xi^{\prime}\right)\right]^{-1} T,
$$

where $\xi^{\prime}$ ranges from $\xi_{\text {in }}^{\prime}$ to $\xi_{\text {out }}^{\prime}$. The code integrates the resultant blackbody spectrum over the surface area from angle $\xi_{\text {in }}$ to $\xi_{\text {out }}$. The area filling factor of the blackbody emission is set by the size, temperature, and luminosity of the torus, with the latter derived from the IR flux observed at the Earth. Direct observations of the dust emission from the quasar 4C 21.35 (Malmrose et al. 2011) guide the choice of these physical parameters. In this $\gamma$-ray bright object, the flux of the dust emission is almost entirely from a single-temperature blackbody $(T \approx 1200 \mathrm{~K})$ and has a value of 0.22 times the flux of the accretion disk that provides most of the optical-ultraviolet luminosity. In the case of $4 \mathrm{C} 21.35$, the area filling factor of the dust emission is $\sim 0.6 \mathrm{pc}^{2} /\left(r_{t} R_{t}\right)$ and $R_{t} \approx 0.22 r_{t}$.

The code calculates the synchrotron and first-order SSC emission generated by the plasma in the MD at each time step (see above). Since the cross-sectional size of the MD is not well specified by gas dynamical theory, it is left as a free parameter (see Table 1). The plasma decelerates from a high Lorentz factor to $\Gamma_{d} \approx 1.2$ as it crosses the MD shock front, hence the shock greatly magnifies the magnetic field and electron density relative to the values in other cells. The seed photons from the MD arrive at each cell with a time delay that depends on the distance of the cell from the MD. The blueshift $\delta_{\mathrm{MD}}$ of the MD photons in the frame of the plasma in the cell depends on the angle between the cell's line of sight to the MD and the velocity vector of the plasma relative to that of the MD plasma, with the latter assumed to be $\beta_{\mathrm{MD}}=1 / 3$ in the $\hat{\mathbf{z}}$ direction. The flux then depends on both the inverse square of the distance of the cell from the MD and the relativistic beaming factor $\delta_{\mathrm{MD}}^{2+\alpha}$, where the dependence of the flux density $F_{v}$ on frequency $v$ is $F_{v} \propto v^{-\alpha}$. (Since $\alpha$ can change with frequency, its value is determined by the code from the emission calculation.) The synchrotron flux received from the MD also depends on the angle $\psi_{\mathrm{MD}}$ that the MD magnetic field subtends to the aberrated line of sight between the cell and the MD, $F_{v} \propto\left(\sin \psi_{\mathrm{MD}}\right)^{1+\alpha}$. The synchrotron self-absorption optical depth within the MD for radiation that will intersect the cell is proportional to $\left(\sin \psi_{\mathrm{MD}}\right)^{1.5+\alpha}$. The current version of the code approximates the optical depth of synchrotron selfabsorption in intervening cells by multiplying the absorption coefficient of the cell by the distance between the MD and the cell. 


\subsection{Linear Polarization}

The current version of the TEMZ code calculates the linear polarization for each cell in a simple manner. For an optically thin cell, the degree of polarization depends on the (frequencydependent) spectral index, $\Pi_{\text {cell }}=(\alpha+1.0) /[\alpha+(5.0 / 3.0)]$, with EVPA perpendicular to the direction of the magnetic field as projected on the sky. In the optically thick case $\Pi_{\text {cell }}=$ $3 /(12 \alpha+19)$, and the EVPA is along the projected direction of the magnetic field (Pacholczyk 1970). These relations are valid because the magnetic field is treated as uniform within each cell. The code calculates the value of $\chi_{\text {cell }}$, aberrated by relativistic motion, following the formulation of Lyutikov et al. (2005). From the flux density of the cell $F_{v \text {,cell }}$ determined as in Section 2.2, the Stokes parameters are then set as $Q_{s \text {,cell }}=$ $F_{v, \text { cell }} \cos 2 \chi_{\text {cell }}$ and $U_{s \text {,cell }}=F_{v \text {,cell }} \sin 2 \chi_{\text {cell }}$. The code sums $Q_{s, \text { cell }}$ and $U_{s \text {,cell }}$ over all of the cells to determine the integrated degree of polarization $\Pi=\left(Q_{s}^{2}+U_{s}^{2}\right)^{1 / 2} / F_{v}$ and position angle $\chi=0.5 \tan ^{-1}\left(U_{s} / Q_{s}\right)$.

\section{TESTING OF THE NUMERICAL CODE}

Because of the complexity of the algorithms needed to carry out the calculations, the author has tested the output of the code in a variety of ways. The first set of tests involved comparison of the synchrotron and IC spectrum computed for single cells, with standard analytical expressions for a cylindrical source with uniform properties (see, e.g., Pacholczyk 1970). The position angle of linear polarization of the synchrotron emission was tested for different directions of uniform magnetic fields relative to the shock front and to the line of sight. The difference in degree and position angle of polarization with shock compression was compared with the case of no compression. A print-out of the evolution of the electron energy distribution of a given parcel of plasma provided a check on the numerical handling of how radiative energy losses affect the distribution. The code passed all of these basic tests.

Testing of the full multi-zone code involved checks on the various time delays for propagation of electromagnetic waves and advection of plasma downstream of the shock. The introduction of a very high-amplitude pulse of relativistic electron injection (i.e., a sudden increase in $Q_{0}$ ) over a few time steps facilitated comparison of the time-dependent output of the code with the expected behavior. Sample results are displayed in Figure 2. Here we see that, for $\theta_{\mathrm{los}} \approx 0$, the induced optical synchrotron and EC-Dust flux rises steeply as the pulse crosses the ring of computational cells at the upstream end of the shock, then declines more gradually as the pulse crosses smaller rings of cells. (The electrons that produce $\gamma$-ray and optical photons lose energy rapidly, hence radiation at these frequencies is confined to a thin layer close to the shock.) The SSC-MD flux increases modestly at first before a rapid flare occurs as the pulse crosses the MD, creating a sudden increase in seed photon flux.

\section{RESULTS OF SAMPLE NUMERICAL SIMULATIONS}

Figure 3 presents samples of light curves, polarization versus time, and SEDs for two of the TEMZ simulations. The parameters were selected to be similar to those of two blazars, BL Lacertae and PKS 1510-089, as derived from observations (e.g., Jorstad et al. 2005; Marscher et al. 2008, 2010). They are not intended, however, as actual fits to any data. In fact, because of the randomness inherent in the model, close fits to observational data cannot be attained. Rather, future success of the model will depend on comparison of the statistical characteristics of the simulated results with those of the data.

The BL Lac-like simulation bears a resemblance to the observed flux and polarization versus time curves of this blazar presented in Marscher (2013). The running mean of the $\gamma$-ray flux level increases with time, while on short timescales the flux varies erratically. The fluxes at lower frequencies rise and fall roughly together, although with some cross-frequency delays in maxima and minima. The fluctuations about the running mean at optical and X-ray frequencies occur on similar timescales as at $\gamma$-ray energies, but with lower amplitudes, while the variations at $\lambda 1 \mathrm{~mm}(230 \mathrm{GHz})$ are quite smooth owing to the larger volume occupied by electrons that emit at this wavelength relative to those responsible for the optical emission. There are "orphan" (i.e., with no counterpart at the other wavebands) $\gamma$ ray flares (e.g., at $t \sim 30$ days) and optical flares without $\gamma$-ray counterparts (e.g., at $t \sim 46$ days). The higher amplitude of the $\gamma$-ray variations relative to those at optical frequencies results both from the somewhat higher (factor of $\sim 2-3$ ) energies - and therefore shorter radiative lifetimes - of electrons that produce $\gamma$-rays than those that produce optical synchrotron radiation, as well as variations in seed photon energy density throughout the emission region owing to changing conditions in the MD. Since IR emission by hot dust has yet to be confirmed in a BL Lac object, the simulation includes no contribution to the seed photon field from this component.

The simulation of a blazar similar to the quasar PKS 1510-089 (bottom row of Figure 3) produces SSC-MD $\gamma$ ray and synchrotron optical light curves that are quite similar, although not identical, in profile. The EC-Dust $\gamma$-ray flux varies more erratically because the electrons that scatter the dustemitted photons to $\gamma$-ray energies are near the highest energies generated by the shock, and therefore occupy a small fraction of the total volume. Such high energies result from the lower mean frequency of the seed photons from the dusty torus relative to the synchrotron and SSC emission from the MD. [Note that the calculation of the seed photon field includes integration over the torus (see Section 2.4), rather than the crude (unless the opening angle of the torus is very large) approximation that the dust-emitted photons are isotropic in the quasar rest frame at the position of the jet plasma, as approximated, for example, by Ghisellini \& Tavecchio 2009.] The X-ray and $\lambda 1 \mathrm{~mm}$ light curves are well-correlated, as observed (e.g., Marscher et al. 2010), although they do not follow each other completely.

Many of the flares have quite sharp peaks, with rises and decays that are approximately linear or exponential. This is a common characteristic of blazar light curves (e.g., Chatterjee et al. 2012) that is difficult for many models to reproduce. The timescales of the variations, especially at optical and $\gamma$-ray frequencies, can be extremely short, as anticipated by Marscher \& Jorstad (2010) and Narayan \& Piran (2012). This is a result of the combination of (1) the small volume filling factor of production of the highest energy electrons, (2) the short radiative lifetimes of these electrons, (3) the high Lorentz factor of the laminar flow, and (4) the turbulent peculiar velocities $\beta_{t}$, which can increase the Doppler factor by as much as a factor of $\left(1-\beta_{t}^{2}\right)^{-1 / 2}$

The dependence of the volume filling factor on frequency leads to two additional features of the model that compare well with observations (see Jorstad et al. 2007; Marscher \& Jorstad 2010; Wehrle et al. 2012; Jorstad et al. 2013). The time-averaged degree of linear polarization increases, and the timescale of variability of both the degree and position angle of polarization 

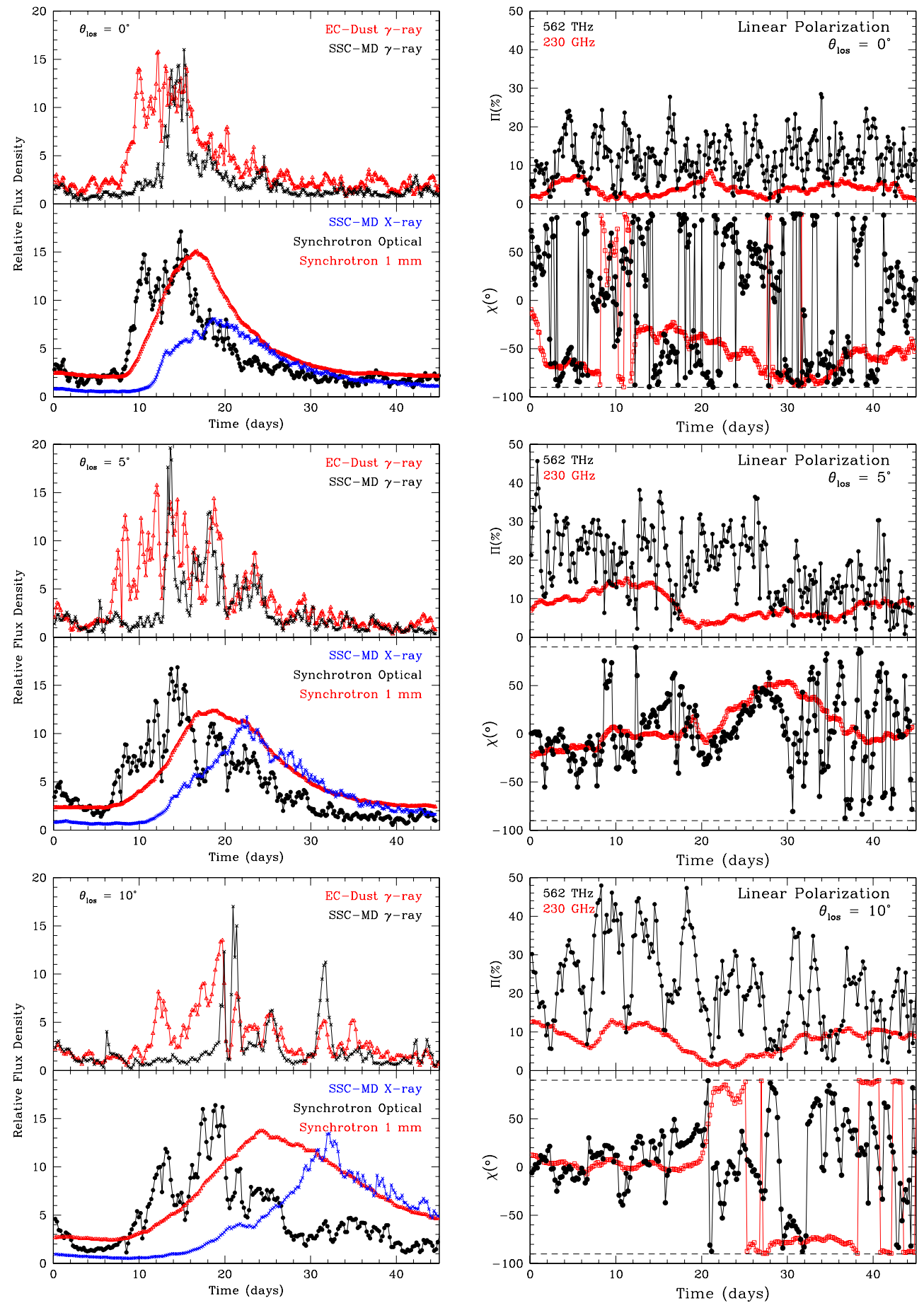

Figure 2. Light curves (left) and polarization vs. time (right) simulated with the TEMZ code for the test case of a sudden, short increase in the injection rate of relativistic electrons, as described in the text. The overall profiles reflect the effect of the chosen geometry (see Figure 1) plus, on short timescales, the effect of turbulence. The three cases displayed correspond to different viewing angles $\theta_{\text {los }}$. Note that polarization position angle $\chi=+90^{\circ}$ is the same as $-90^{\circ}$. The projected position angle of the jet axis is $0^{\circ}$.

decreases while the amplitude of variability increases, with frequency. Furthermore, breaks in the spectrum by more than by 0.5 (expected from radiative losses in a single emission zone; e.g., Marscher \& Gear 1985) appear at IR and $\gamma$-ray frequencies.

As with the light curves, the polarization versus time curves in Figure 3 (middle panels) also exhibit a combination of systematic and erratic behavior. As expected, the optical polarization (represented in the figure by $562 \mathrm{THz}$, or $534 \mathrm{~nm}$, which is within the $V$ band) ranges from close to zero up to tens of percent for the
BL-Lac-like simulation and up to nearly $20 \%$ for the quasar-like simulation, similar to the observed range (see Marscher et al. 2010; Marscher 2013, to compare with data). The mean EVPA fluctuates about the jet direction $\left(0^{\circ}\right.$ in the simulations), as is generally observed in BL Lac. Apparent rotations of the polarization vector occur, although a larger number of simulations is needed to determine whether they resemble the variety of such rotations that are observed in blazars (e.g., Marscher et al. 2008; Larionov et al. 2008, 2013; Abdo et al. 2010). Such rotations are 

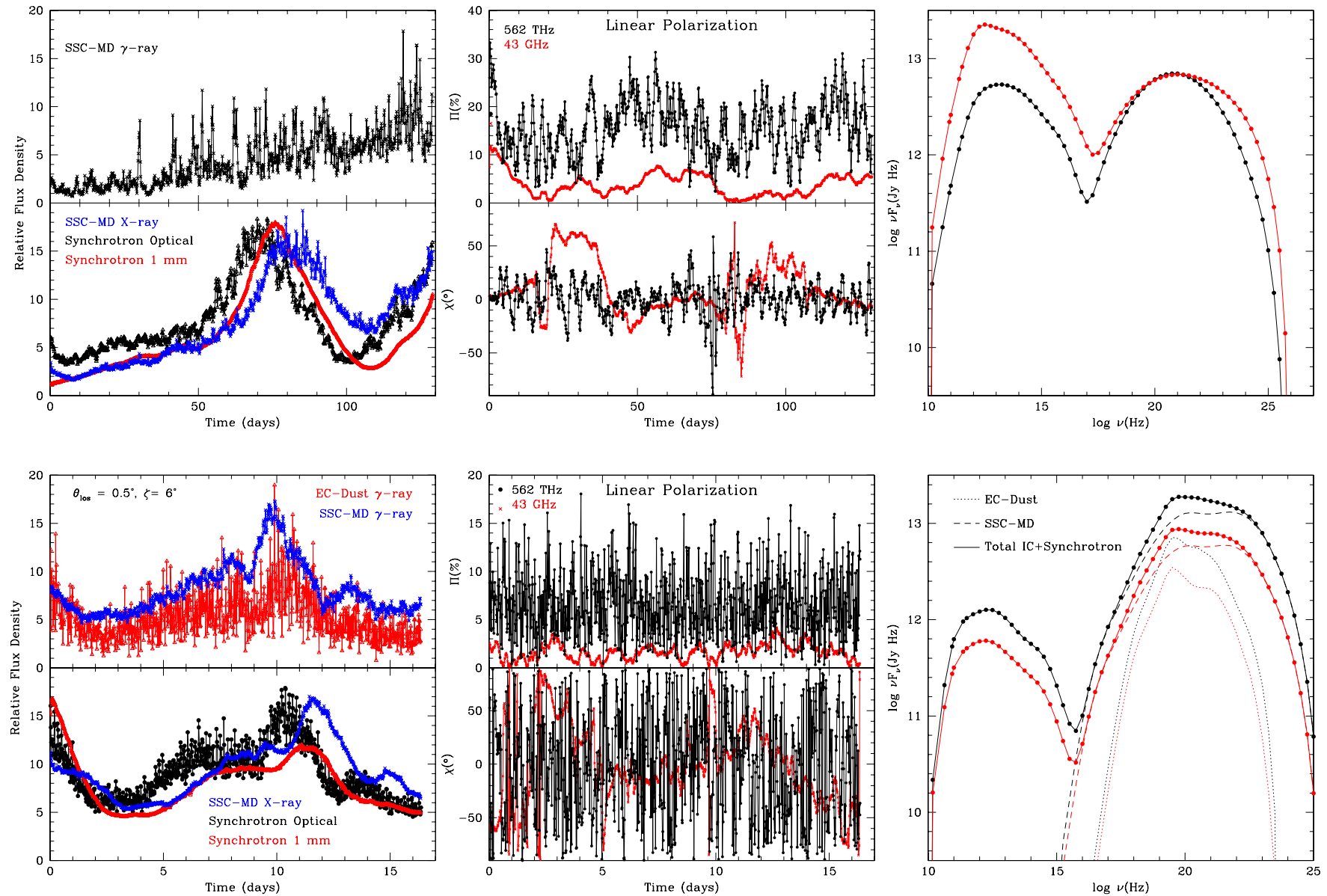

Figure 3. Sample light curves (left), polarization vs. time (middle), and SED at two times (right) from TEMZ simulations. Flux levels are scaled arbitrarily so that the shape of each light curve is apparent. Top row: parameters selected to be similar to BL Lacertae: $n_{\text {rad }}=7$ (168 cells across the shock front), $z_{\mathrm{MD}}=1.22 \mathrm{pc}$, $Z=0.069, \alpha=0.55, b=1.7, B=0.04 \mathrm{G}, f_{B}=1.0, R_{\text {cell }}=0.003 \mathrm{pc}, \gamma_{\min }=300, \gamma_{\max , \text { high }}=140,000, \gamma_{\max , \text { low }}=7000, \beta_{u}=0.990, \beta_{t}=0.577, \zeta=10^{\circ}$, $\theta_{\text {los }}=7.7, \phi=1.9, A_{\mathrm{MD}}=0.01$, and no significant thermal emission from dust. The SEDs correspond to times of 70.76 days (red) and 107.6 days (black). Temporal resolution is 0.129 days $\left(3.1 \mathrm{hr}\right.$ ) over 1000 time steps. Bottom row: parameters selected to be similar to PKS 1510-089: $n_{\text {rad }}=10$ ( 270 cells across the shock front), $z_{\mathrm{MD}}=1.18 \mathrm{pc}, Z=0.361, \alpha=0.7, b=1.7, B=0.04 \mathrm{G}, f_{B}=1.0, R_{\text {cell }}=0.001 \mathrm{pc}, \gamma_{\min }=1800, \gamma_{\max , \text { high }}=37,500, \gamma_{\max , \text { low }}=5000, \beta_{u}=0.99969$, $\beta_{t}=0.577, \zeta=6^{\circ}, \theta_{\mathrm{los}}=0.5, \phi=0.2, A_{\mathrm{MD}}=1, T_{\text {dust }}=1200 \mathrm{~K}, L_{\text {dust }}=1 \times 10^{46} \mathrm{erg} \mathrm{s}^{-1}, r_{\text {dust }}=3.0 \mathrm{pc}$, and $R_{\text {dust }}=0.8$ pc. The SEDs correspond to times of 3.0 days (red) and 10.0 days (black). The temporal resolution is 0.0159 days (23 minutes) over 1027 time steps.

common when the degree of polarization is low (see also Jones 1988; Marscher et al. 1992). If the randomness in the magnetic field directions causes the polarization integrated over $\mathcal{N}-\mathcal{M}$ of the cells to cancel, with $\mathcal{N} \gg \mathcal{M}$, the net polarization is low and determined by the other $\mathcal{M}$ cells. Since a small number of cells is involved, time variations can occur that resemble rotations of the polarization vector. However, such apparent rotations are unlikely to explain the systematic optical EVPA rotations reported in BL Lac (Marscher et al. 2008) and PKS 1510-089 (Marscher et al. 2010). Both of these observed events occurred prior to the time when the emitting plasma crossed the core, and hence took place upstream of the location where the TEMZ calculations apply. Despite the slower variations of polarization at longer wavelengths, rotations of the polarization vector still occur at millimeter wavelengths both in the simulations and in observations (e.g., Larionov et al. 2008).

Both the degree and position angle of the quasar-like simulation vary more erratically than is typically observed in PKS 1510-089 (e.g., Marscher et al. 2010). This discrepancy is likely the result of the lack of continuity in the magnetic field vector across cells in the direction transverse to the jet axis in the current version of the TEMZ code (see Section 2). Actual jets may also contain an ordered component of the magnetic field in addition to the turbulent component. If so, the very short timescale variations in polarization will have lower amplitudes.

Figure 4 presents sample total $(I)$ and polarized $(P)$ intensity images at $43 \mathrm{GHz}$ generated at a particular time of the quasarlike simulation. These images serve as representations of the brightness and polarization distribution across the source, as well as an indication of the ability of VLBI observations to test the TEMZ model. In order to generate the images, a rectangular grid of pixels is set up, with the intensity from all of the cells that lie along the line of sight represented by each pixel summed (vectorially for a $P$ image) to obtain the intensity of the pixel. The pixelated image is then convolved with a circular Gaussian restoring beam (i.e., a point-spread function). The image at the top of the figure is convolved with a small beam to reveal the underlying intensity pattern, while the image at the bottom has a FWHM resolution equal to the diameter of the jet cross-section. The latter is typical of the angular resolution of the longest baselines of a millimeter-wave VLBI observation with nearly Earth-diameter maximum separations of antennas. The addition of one or more space-based antennas is therefore needed in order to determine whether a given blazar possesses the brightness and polarization structure predicted by the model. 

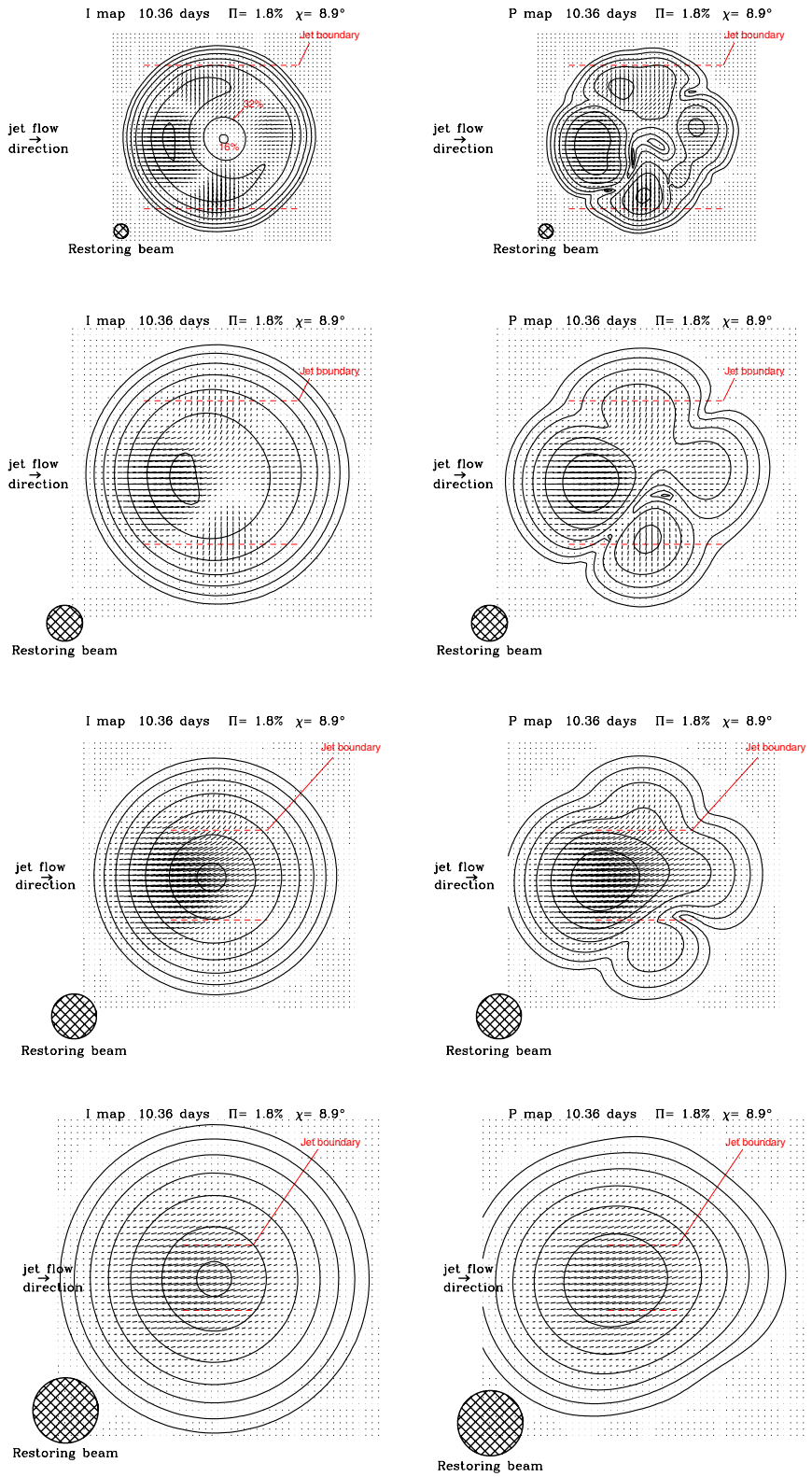

Figure 4. Simulated total (left) and polarized (right) intensity images at $43 \mathrm{GHz}$ of the section (denoted by dashed lines) of a blazar jet whose emission is calculated by the TEMZ code. Contour levels correspond to $2 \%, 4 \%, 8 \%, 16 \%$, $32 \%$, and $64 \%$ of the peak intensity; for the total intensity images, an additional $95 \%$ contour is added to define the location of the peak. (Note that the two contours near the center of the top left image represent decreases in intensity, to $32 \%$ and $16 \%$, as marked.) The line segments represent the orientation of the polarization electric vector, with lengths proportional to the polarized intensity. All images are from time 10.36 days of Figure 3 (bottom panels). The intensity is smoothed with a circular Gaussian restoring beam (point-spread function), with progressively decreasing FWHM diameter from top to bottom, as indicated to the bottom left of each image.

(A color version of this figure is available in the online journal.)

Comparison of the top right and bottom right images in Figure 4 demonstrates that the low net polarization at $43 \mathrm{GHz}$ is caused by cancellation of cross-polarized sections, which is nearly complete for very small viewing angles because of the underlying symmetry of the conical shock structure. The deviation from perfect symmetry between the top and bottom halves of the images is caused by the random elements in the TEMZ model. Except for this effect, which is small at $43 \mathrm{GHz}$ owing to the large number of cells that emit at this frequency, the polarized intensity structure is similar to that calculated and displayed by Cawthorne (2006), Nalewajko (2009), and Cawthorne et al. (2013). These authors consider the somewhat different case of a standing shock compressing a magnetic field that is completely chaotic upstream of the shock.

\section{CONCLUSIONS}

The many-zone emission model provides much more realistic emission calculations than do schemes involving a very small number of zones. The TEMZ model, improved in ways discussed below, therefore holds some promise in explaining the diversity of blazar variability in multi-waveband flux, polarization, and structure. While this may seem to be at the expense of complexity, the number of free parameters, which are listed in Table 1, is similar to that of single-zone models, with many of the parameters $\left(Z, \alpha, b, \beta_{u}, \theta_{\text {los }}, \phi, T_{\text {dust }}\right.$, and $\left.L_{\text {dust }}\right)$ specified by observations. Furthermore, instead of fitting the data to SEDs measured at a small number of times, the results produced by the TEMZ code can be compared with the time evolution of the SED as well as the characteristics of the light curves, degree and position angle of polarization versus time, and total as well as polarized intensity images of blazars. The author plans to explore how these characteristics as produced by the simulations depend on the adjustable parameters. This will include timing analyses of the simulated flux and polarization curves so that the cross-frequency correlations and PSDs can be compared with those observed in blazars. The results displayed in Figure 3 and discussed in Section 4 resemble observations of blazars well enough to warrant such a study.

There is, on the other hand, one aspect of the simulations that conflicts with the data: the ratio of $\gamma$-ray to X-ray luminosity (right-most panels of Figure 3) is not as high as observed during major outbursts of some of the most prominent $\gamma$-ray bright quasars. This problem with the simulations was pointed out by Wehrle et al. (2012), who used the TEMZ code to fit the SEDs of the quasar 3C 454.3 during a major outburst. The over-production of X-rays in the model is caused by the strong synchrotron emission from the MD at far-IR wavelengths, as viewed in the rest frame of the plasma in the other cells. Perhaps a more refined treatment of the MD, based on magnetohydrodynamic (MHD) simulations (see below), will solve this problem. On the other hand, a thermal spectrum of seed photons, which declines sharply (as $v^{2}$ ) toward low frequencies, does not produce an X-ray excess as long as $\gamma_{0, \text { min }} \gtrsim 100$. The high $\gamma$-ray to X-ray flux ratio is one reason why seed photons from thermal emission from broad emission-line clouds and a dusty torus is favored by many authors (e.g., Ghisellini \& Tavecchio 2009). However, at distances $\gtrsim 3$ pc from the central engine, the seed photon field from these regions is too weak to explain the high $\gamma$-ray luminosity. If, instead, some stray molecular clouds or dense, ionized clouds are located along the periphery of the jet, it is possible that they could produce a sufficient density of seed photons to explain the $\gamma$-ray emission from IC scattering (Isler et al. 2013; León-Tavares et al. 2013). The author plans to add such a source of seed photons to the code. Also, in order to simulate blazars with ratios of $\gamma$-ray to IR luminosities closer to unity, development of a full SSC and SSC-C calculation, with seed photons from all of the cells (calculated in retarded time), is underway.

It is likely that, in many blazars, other physical processes besides a single standing shock are responsible for energizing electrons to induce flares as well as more quiescent emission. In fact, there is evidence in a number of blazars for multiple 
standing features that could correspond to oblique or conical shocks (e.g., Jorstad et al. 2005). The TEMZ model could be applied separately to emission from any such stationary structures, although if there is a non-conical shock, the code would need to be altered accordingly. Moving shocks in a jet filled with turbulent plasma (Marscher et al. 1992) are possible as well, especially when a very bright superluminal knot is observed to propagate down the jet. Magnetic reconnections have been proposed as an alternative to shocks as a primary process for accelerating electrons, causing relativistic bulk motions of plasma, and therefore generating flares (e.g., Giannios et al. 2009). The TEMZ code can potentially be modified to incorporate this scenario after studies of relativistic reconnection elucidate how the magnetic field topography and electron energy distribution vary with time and position.

While randomness in the magnetic field direction in different turbulent cells can cause observed rotations in the linear polarization vector, it probably does not explain the systematic rotations observed in some blazars. The conclusion that such events occur before a disturbance in the jet reaches the standing shock implies that the variable emission described in the TEMZ code does not cover all of the events in the light curves of blazars. This is also true at lower frequencies where much of the emission comes from regions farther downstream in the jet, and even in some cases at $\gamma$-ray energies where observations indicate that flares can take place many parsecs from the core (Agudo et al. 2011a, 2011b). Nevertheless, there is strong evidence that most of the multi-waveband outbursts in blazars are associated with emission in the core region treated in the TEMZ model (Marscher et al. 2012).

Despite the promising results presented here, more realistic simulations are needed to confront the model with data in a serious way. The geometry and physical properties of the standing shock and MD system are simplified in the current version of the TEMZ code. Ideally, they should be determined instead by relativistic MHD simulations. The energization of relativistic electrons is also treated in an ad hoc manner, as is the approximation to turbulence. Furthermore, the fluctuations in electron energy density are forced to agree with the observed PSD of blazar flux variations, while they ideally should be dictated by successful simulations of flow from the accreting $\mathrm{BH}$ system into the jet. The author plans to collaborate with other groups working in these areas to combine the TEMZ emission code with detailed calculations of the dynamics and plasma physics of relativistic jets in order to produce more realistic simulations of emission from blazar jets.

The author thanks R. Chatterjee for providing his code for producing random fluctuations that obey the statistics of a powerlaw PSD; S. Jorstad, M. Joshi, J. L. Gómez, N. MacDonald, M. Baring, and M. Böttcher for discussions that were useful in the development of the model (and the first four plus M. Malmrose for critical readings of a draft version of the manuscript); M. Valdez for scrutinizing, and identifying some flaws in, the numerical code and for translating it to $\mathrm{C}++$; and J. L. Gómez for providing visualization software that aided in testing of the results. He also thanks Professor Stefan Wagner for his hospitality during a visit to the University of Heidelberg in 1998 when this study was first conceived. Funding of this research included NASA grants NNX10AO59G, NNX11AQ03G, and NNX12AO79G, and NSF grant AST-0907893. This work benefited from discussion within International Team Collaboration 160 sponsored by the International Space Science Institute (ISSI) in Switzerland.

\section{REFERENCES}

Abdo, A. A., Ackermann, M., Ajello, M., et al. 2010, Natur, 463, 919 Abdo, A. A., Ackermann, M., Ajello, M., et al. 2011, ApJL, 733, L26 Agudo, I., Jorstad, S. G., Marscher, A. P., et al. 2011a, ApJL, 726, L13 Agudo, I., Marscher, A. P., Jorstad, S. G., et al. 2011b, ApJL, 735, L10 Burn, B. J. 1966, MNRAS, 133, 67

Cawthorne, T. V. 2006, MNRAS, 367, 851

Cawthorne, T. V., \& Cobb, W. K. 1990, ApJ, 350, 536

Cawthorne, T. V., Jorstad, S. G., \& Marscher, A. P. 2013, ApJ, 772, 14 Chatterjee, R., Jorstad, S. G., Marscher, A. P., et al. 2008, ApJ, 689, 79 Chatterjee, R., Marscher, A. P., Jorstad, S. G., et al. 2009, ApJ, 704, 1689 Chatterjee, R., Bailyn, C. D., Bonning, E. W., et al. 2012, ApJ, 749, 191 Courant, R., \& Friedrich, D. B. 1976, Supersonic Flow and Shock Waves (New York: Springer)

D'Arcangelo, F. D. 2009, PhD thesis, Boston Univ.

Daly, R. A., \& Marscher, A. P. 1987, ApJ, 334, 539

D’Arcangelo, F. D., Marscher, A. P., Jorstad, S. G., et al. 2007, ApJL, 659, L107

Dermer, C. D., \& Menon, G. 2009, High Energy Radiation from Black Holes: Gamma Rays, Cosmic Rays, and Neutrinos (Princeton, NJ: Princeton Univ. Press)

Emmanoulopoulos, D., McHardy, I. M., \& Papadakis, I. E. 2013, MNRAS, 433, 907

Ghisellini, G., \& Tavecchio, F. 2009, MNRAS, 397, 985

Giannios, D., Uzdensky, D. A., \& Begelman, M. C. 2009, MNRAS, 395, L29

Gómez, J. L., Martí, J. M., Marscher, A. P., Ibáñez, J. M., \& Marcaide, J. M. 1995, ApJL, 449, L19

Isler, J. C., Urry, C. M., Coppi, P., et al. 2013, ApJ, 779, 100

Itoh, R., Fukazawa, Y., Tanaka, Y. T., et al. 2013, ApJL, 768, L24

Jones, T. W. 1988, ApJ, 332, 678

Jorstad, S. G., Marscher, A. P., Lister, M. L., et al. 2005, AJ, 130, 1418

Jorstad, S. G., Marscher, A. P., Smith, P. S., et al. 2013, ApJ, 773, 147

Jorstad, S. G., Marscher, A. P., Stevens, J. A., et al. 2007, AJ, 134, 799

Joshi, M., \& Böttcher, M. 2011, ApJ, 727, 21

Kardashev, N. S. 1962, AZh, 39, 393

Larionov, V. M., Jorstad, S. G., Marscher, A. P., et al. 2008, A\&A, 492, 389

Larionov, V. M., Jorstad, S. G., Marscher, A. P., et al. 2013, ApJ, 768, 40

León-Tavares, J., Chavushyan, V., Patiño-Álvarez, V., et al. 2013, ApJL, 763, L36

Lind, K. R., \& Blandford, R. D. 1985, ApJ, 295, 358

Lyutikov, M., Pariev, V. I., \& Blandford, R. D. 2003, ApJ, 597, 998

Lyutikov, M., Pariev, V. I., \& Gabuzda, D. C. 2005, MNRAS, 360, 869

Malmrose, M., Marscher, A. P., Jorstad, S. G., Nikutta, R., \& Elitzur, M. 2011, ApJ, 732, 116

Marscher, A. P. 2013, in eConf C121028, 2012 Fermi Symp., ed. N. Omodel, T. Brandt, \& C. Wilson-Hodge (arXiv:1304.2064)

Marscher, A. P., Jorstad, S. G., D’Arcangelo, F. D., et al. 2008, Natur, 452, 966

Marscher, A. P., Jorstad, S. G., Larionov, V. M., et al. 2010, ApJL, 710, L126

Marscher, A. P., \& Gear, W. K. 1985, ApJ, 298, 114

Marscher, A. P., Gear, W. K., \& Travis, J. P. 1992, in Variability of Blazars, ed. E. Valtaoja \& M. Valtonen (Cambridge: Cambridge Univ. Press), 85

Marscher, A. P., \& Jorstad, S. G. 2010, in Fermi Meets Jansky-AGN at Radio and Gamma-Rays, ed. T. Savolainen, E. Ros, R. W. Porcas, \& J. A. Zensus (Bonn: Max-Planck-Institut für Radioastronomie), 171

Marscher, A. P., Jorstad, S. G., Agudo, I., MacDonald, N. R., \& Scott, T. L. 2012, in eConf C1111101, Fermi \& Jansky: Our Evolving Understanding of AGN, ed. R. Ojha, D. Thompson, \& C. D. Dermer (arXiv:1204.6707)

Nalewajko, K. 2009, MNRAS, 395, 524

Narayan, R., \& Piran, T. 2012, MNRAS, 420, 604

Pacholczyk, A. G. 1970, Radio Astrophysics (San Francisco: Freeman)

Pushkarev, A. B., Gabuzda, D. C., Vetukhnovskaya, Yu. N., \& Yakimov, V. E. 2005, MNRAS, 356, 859

Pushkarev, A. B., Lister, M. L., Kovalev, Y. Y., \& Savolainen, T. 2012, in eConf C1111101, Fermi \& Jansky: Our Evolving Understanding of AGN, ed. R. Ojha, D. Thompson, \& C. D. Dermer (arXiv:1205.0659)

Sari, R., \& Esin, A. A. 2001, ApJ, 548, 787

Summerlin, E. J., \& Baring, M. G. 2012, ApJ, 745, 63

Timmer, J., \& König, M. 1995, A\&A, 300, 707

Uttley, P., McHardy, I. M., \& Vaughan, S. 2005, MNRAS, 359, 345

Wehrle, A. E., Marscher, A. P., Jorstad, S. G., et al. 2012, ApJ, 758, 72 